\title{
Chain Length Scaling of Protein Folding Time
}

\section{Citation}

Gutin, A. M., V. I. Abkevich, and E. I. Shakhnovich. 1996. "Chain Length Scaling of Protein Folding Time." Physical Review Letters 77 (27): 5433-36. https://doi.org/10.1103/physrevlett.77.5433.

\section{Permanent link}

http://nrs.harvard.edu/urn-3:HUL.InstRepos:41417268

\section{Terms of Use}

This article was downloaded from Harvard University's DASH repository, and is made available under the terms and conditions applicable to Other Posted Material, as set forth at http:// nrs.harvard.edu/urn-3:HUL.InstRepos:dash.current.terms-of-use\#LAA

\section{Share Your Story}

The Harvard community has made this article openly available.

Please share how this access benefits you. Submit a story.

Accessibility 


\title{
Chain length scaling of protein folding time.
}

\author{
A. M. Gutin, V. I. Abkevich, and E. I. Shakhnovich \\ Harvard University, Department of Chemistry \\ 12 Oxford Street, Cambridge MA 02138
}

\begin{abstract}
Folding of protein-like heteropolymers into unique 3D structures is investigated using Monte Carlo simulations on a cubic lattice. We found that folding time of chains of length $N$ scales as $N^{\lambda}$ at temperature of fastest folding. For chains with random sequences of monomers $\lambda \approx 6$, and for chains with sequences designed to provide a pronounced minimum of energy to their ground state conformation $\lambda \approx 4$. Folding at low temperatures exhibits an Arrheniuslike behavior with the energy barrier $E_{b} \approx \phi\left|E_{n}\right|$, where $E_{n}$ is the energy of the native conformation. $\phi \approx 0.18$ both for random and designed sequences. PACS numbers: 87.10.+e, 82.20.Db, 05.70.Fh, 64.60.Cn
\end{abstract}

Typeset using REVTEX 
The number of all possible conformation (shapes) of a protein of $N$ amino acids scales as $z^{N}$ where $z$ is the number of conformations per one amino acid residue. Thus, random search in the conformational space for the native, biologically active shape would require folding time $t \sim z^{N}$ which is unrealistic for a typical protein with $N \sim 100$ (Levinthal paradox). In fact, real dynamics of a protein is far from random search. The native structure is believed to represent a pronounced minimum of energy 11 , so that folding process is driven by decrease in energy (opposed by entropy decrease). Moreover, it is clear that folding time should strongly depend on folding conditions. At very high temperature all conformations are equally favorable and folding time should indeed be close to Levinthal's estimate $z^{N}$. At very low temperature one should expect Arrhenius-like slowing-down caused by energy barrier(s) on a folding pathway. Thus, there should be some finite temperature optimal for folding in the sense that folding is fastest at this temperature. Such temperature dependence of the folding time was observed indeed in a number of computer simulations for different models [5] 8 .

Much less is known about length dependence of the folding time. It is clear that folding time should grow when chain length increases but the actual dependence is not known. Though there are some attempts to estimate the folding time from scaling and other simple arguments [9 11], these estimates are based on phenomenological, rather than microscopic, considerations. Experimental data are not readily available to elucidate this important point. The problem here is that folding can involve some kinetic events which are specific to a given protein, such as isomerisation of prolines and formation of disulphide bonds. In addition, large proteins typically consist of few domains with independent, to some extent, folding.

In the present paper we study the length dependence of folding rate using Monte Carlo simulation of folding on a cubic lattice. Despite its simplicity and computational efficiency, this model proved useful in elucidating such crucial, experimentally observed, features of protein folding as cooperativity [12 [14], nucleation mechanism [15]17] and parallel pathways 18, 4, 6, 6], 8 
Both chains with random sequences of amino acids and chains with sequences designed to provide a pronounced energy minimum for a particular compact conformation [3] are analyzed and compared.

Protein chain is modeled as a self-avoiding walk on an infinite cubic lattice [6]. Energy of a given conformation of a chain is given by

$$
H=\sum_{i<j} U\left(a_{i}, a_{j}\right) \Delta_{i j}
$$

where $\Delta_{i j}=1$ if monomers $i$ and $j$ are in contact and $\Delta_{i j}=0$ otherwise. Monomers $i$ and $j$ are considered to be in contact if they are separated by one lattice bond and $|i-j| \neq 1$. Sequence of amino acids is given by $a_{i}$ with $i=1, \ldots, N$, so that $a_{i}$ can take 20 different values corresponding to 20 natural amino acids. Finally, $U(a, b)$ is the energy of a contact between amino acids $a$ and $b$. In this work we use interaction matrix $U(a, b)$ obtained in Ref. [19]. from statistical analysis of protein structures. A number of previous studies suggest [15,17] that the results do not depend on a particular choice of interaction matrix. Dynamics of a chain is modeled by a standard Monte Carlo procedure for a cubic lattice [20].

First, we studied folding of chains with random sequences of monomers. 5 random sequences were generated for each chain length in the range from 10 to 50. A long Monte Carlo simulation was performed for each of these sequences, to identify the conformation with the lowest energy. Fig.1 shows the lowest energy conformation for one of random chains of 40 monomers. In order to make sure that there are no conformations with lower energies, additional 10 runs starting from different unfolded conformations were performed for each sequence. In each of these runs a chain folded to the putative lowest energy conformation, and no conformation with lower energy was encountered.

From 5 random sequences generated for the same chain length, we selected one sequence which folding rate was the fastest. Then for this sequence we studied folding in a range of temperatures to determine the temperature, at which folding rate was fastest. Further, at this temperature, we made a more precise estimate of the mean first passage time (MFPT) to the lowest energy conformation by averaging over 50 runs starting from different unfolded 
conformations. The MFPT for all studied chain lengths is shown in Fig.2. It is seen that folding time grows with chain length, and the dependence can be well described by a powerlaw, that is $t \sim N^{\lambda}$ with $\lambda \approx 6$.

Next, we studied the length dependence of folding rate for sequences designed to have their native conformations as pronounced energy minimum. Such sequences are more likely to exhibit protein-like behavior [12]. To this end, each of the lowest energy conformations found previously for random sequences was used as a target conformation for sequence design. The design procedure is described in detail elsewhere [3, 14, 21]. We did not study random sequences longer than 50 units, while it is feasible to study folding rate of designed sequences up to 100 units long [12]. To this end we generated random compact conformations to serve as target structures for sequence design of longer sequences (more than 50 units in length). Additional condition was applied that variation of energies of native contacts is low enough, in order to eliminate multi-domain behavior for longer sequences [21]. In this way 5 sequences were generated for each of studied chain length, and the sequence with the fastest folding was selected. Length dependence of the folding time at optimal temperature for the designed sequences is presented in Fig.2 for chain lengths ranging from 10 to 100. It can be seen that for short chains folding time of designed sequences is about the same as that of random sequences. For longer chains, though, folding of designed sequences is much faster than folding of random sequences. Overall, length dependence of the folding time for designed sequences is well described by a power law $t \sim N^{\lambda}$ with $\lambda \approx 4$.

For comparison, we also studied the model introduced by Go and coworkers [1]. In this model energy of a given conformation is given by

$$
H=-\frac{1}{2} \sum_{i<j} \Delta_{i j}^{N} \Delta_{i j}
$$

where $\Delta_{i j}^{N}=1$ if monomers $i$ and $j$ are in contact in the native conformation and $\Delta_{i j}^{N}=0$ otherwise. In other words, in the Go model all the native interactions are favorable and all non-native interactions do not contribute to the energy. In some sense, the Go model corresponds to ideally designed sequences. 
For the Go model the same conformations as for designed sequences were used as native. Length dependence of the folding time at optimal temperature for the Go model is presented in Fig.2 for chain lengths from 10 to 175. Again, the dependence is fitted very well by a power law $t \sim N^{\lambda}$ with $\lambda \approx 2.7$.

Fig.3 shows the inverse temperature dependence of the folding time for the sequence Fig.3 designed to fold to the native conformation shown in Fig.1. It has a clear minimum at some optimal temperature. All the scaling dependencies for the folding rate discussed above were obtained at the conditions corresponding, for each sequence, to such an optimal temperature. What happens at lower temperatures? It is clear from Fig.3 that at low temperatures logarithm of folding time depends linearly on inverse temperature exhibiting an Arrheniuslike behavior $t \sim \exp \left(E_{b} / T\right)$. This suggests that at low temperature folding is an activated process which entails overcoming of an energetic barrier $E_{b}$. Similar dependencies were obtained for all studied chains and the corresponding energy barriers $E_{b}$ were determined for each sequence from the slopes of Arrhenius-like branches of these dependencies. The result is presented in Fig. 4 where $E_{b}$ is plotted as a function of energy of the native conformation, Fig.4 $E_{n}$. It can be seen that energy of the low-temperature barrier scales linearly with energy of the native conformation with coefficient $\phi \approx 0.18$. Surprisingly, all the data for random and designed sequences and for the Go model fall on the same line suggesting a universal scaling behavior for the low-temperature energy barrier. This observation can be explained as follows.

The main reason that folding is slow at low temperatures is that the chain gets stuck in some low energy misfolded conformations which needs to be unfolded before chain proceeds further. In the case of random sequences such trapped misfolded conformations are typically very different in structure from the native conformation [22]. Nevertheless, the energy of the misfolded traps is expected to be close to the energy of the native conformation $E_{n}$ [23]. In the case of designed sequences and Go model the native state has much lower energy than any conformation structurally unrelated to the native state. Therefore, for designed sequences, low-energy conformations serving as kinetic traps, have considerable structural 
similarity with the native state. As a result, the energy of deepest kinetic traps are again close to $E_{n}$. In order to escape from such a misfolded state a chain has to break some part of the misfolded conformation. Our simulations suggest that both random and designed chains on a cubic lattice have to rearrange a fraction $\phi$ of the whole structure (independently of its size), in order to escape from a trap. We should note though that the actual estimate of the number of bonds to be broken to escape from the native state may depend on lattice and dynamic algorithm used (e.g. move set). We cannot exclude that this barrier will be dramatically diminished in off-lattice model, so that low-temperature behavior in off-lattice model may be significantly different.

As we pointed out above, the direct comparison between our main result and experiments on real proteins is a challenging task for experimentalists. This will require careful choice of proteins to study as model systems and conditions at which their folding is fastest. We hope that the presented results will stimulate such experiments.

The major finding of this work is that the chain length dependence of folding rate is relatively weak (much weaker than exponential, suggested by Levinthal argument and some phenomenological estimates [8]). This fact is of fundamental importance since it explains, from the folding perspective, the wide range of lengths of existing proteins (roughly 50-1000 aminoacids). Exponential dependence of folding time on protein length would make folding of longer chains prohibitively slow. Another important application of the present result is that it can provide crucial and delicate test of the existing and future kinetic theories. Phenomenological analysis based on the Random Energy Model (REM) predicts exponential dependence of folding rate on system size at all temperatures [8]. This is in contrast with simulation results and probably points out to inapplicability of the REM (and perhaps meanfield models in general) to tackle kinetic issues. This can be understood from the general perspective that folding transition is cooperative (first-order like). Such transitions follow nucleation mechanism at which the transition state is highly inhomogeneous, consisting of islands of the "new" phase in the sea of "old" phase [24]. Such an inhomogeneous distribution (representing the least-activation path) explains the weak size dependence of the rate of 
first-order transitions. It cannot be described by any global homogeneous order parameter. REM-based phenomenological models [8], or approaches, based on Kramers theory [25], use a global order parameter (the number of native-like contacts, Q) as kinetic reaction coordinate. Such approaches miss the inhomogeneity of the transition state and thus predict exponential size dependence of protein folding rate.

A possible physical explanation of the power law rate dependence, for designed sequences is close to the arguments presented recently by Thirumalai [11]. Since folding is cooperative (first-order like) process [12,13], the transition state is reached when nucleus of the folded conformation is formed [15] 17]. The power-law length scaling of the folding rate implies that nucleus does not grow with chain length. In this case the length dependence of the folding rate appears due to entropic cost of loop closure around the folding nucleus. The free energy of loop closure depends logarithmically on its length [26], and this factor, combined with power-law length dependence of polymer relaxation time [26] apparently translates into the overall power-law dependence of the folding rate.

The difference between random and designed sequences is crucial . First of all we see that designed sequences fold faster at their respective optimal folding temperatures and the difference in folding rates between random and designed sequences becomes more pronounced as chains become longer (due to different exponents in the scaling laws describing length dependencies).

Real proteins must not only reach their native state fast but also be stable in their native conformations. This points out to another crucial difference between random and designed sequences: designed sequences of different lengths are stable in the native state at the temperature of their fastest folding while longer random sequences get less stable at their corresponding fastest folding temperature. (Data not shown).

Finally, we note that while power law scaling fits our data best, the range of chain lengths, which we studied, spanned only about an order of magnitude. This length range is most limited for random sequences; for them we cannot rule out a weak stretched exponential, rather than power length scaling [1]. We believe that microscopic analytical theory of 
protein folding dynamics can give satisfactory answer to the important question of length dependence of protein folding time. It is our hope that presented results will stimulate the development of such theory.

\section{ACKNOWLEDGMENTS}

This work was supported by the David and Lucille Packard Foundation and NIH. Interesting discussions with D.Thirumalai and A.Grosberg are gratefully acknowledged. 


\section{REFERENCES}

[1] H. Taketomi, Y. Ueda, and N. Go, Int. J. Peptide Protein Res. 7, 445 (1975).

[2] R.Goldstein, Z.A. Luthey-Schulten, and P.Wolynes, Proc. Natl. Acad. Sci. USA 89, 4918 (1992).

[3] E. I. Shakhnovich and A. M. Gutin, Proc. Natl. Acad. Sci. USA 90, 7195 (1993).

[4] A. Sali, E. Shakhnovich, and M. Karplus, Nature. 369, 248 (1994).

[5] R. Miller, C. A. Danko, J. Fasolka, A. C. Balazs, H. S. Chan, and K. A. Dill, J. Chem. Phys. 96, 768 (1992).

[6] V. I. Abkevich, A. M. Gutin, and E. I. Shakhnovich, J. Chem. Phys. 101, 5062 (1994).

[7] H. S. Chan and K. A. Dill, J. Chem. Phys. 100, 9238 (1994).

[8] J.D.Bryngelson et al, Proteins, 21, 167 (1995).

[9] E. I. Shakhnovich and A. M. Gutin, Europhys. Lett. 9, 569 (1989).

[10] J. D. Bryngelson and P. G. Wolynes, J. Phys. Chem. 93, 6902 (1989).

[11] D. Thirumalai, J. Phys. I France 5, 1457 (1995).

[12] E. I. Shakhnovich, Phys. Rev. Lett. 72, 3907 (1994).

[13] M.-H.Hao and H.Scheraga, J.Phys.Chem. 98, 4940 (1994).

[14] V. I. Abkevich, A. M. Gutin, and E. I. Shakhnovich, J.Mol.Biol. 252, 460 (1995).

[15] V. I. Abkevich, A. M. Gutin, and E. I. Shakhnovich, Biochemistry, 33, 10026 (1994).

[16] A.Fersht , Proc. Natl. Acad. Sci. USA 92, 10869 (1995).

[17] E. I. Shakhnovich, V. I. Abkevich, and O.B.Ptitsyn, Nature, 379, 96 (1996).

[18] S.E Radford and C.M.Dobson, Philos.Trans.R.Soc. London B 348, 17 (1995) 
[19] S. Miyazawa and R. L. Jernigan, Macromolecules 18, 534 (1985).

[20] P. H. Verdier and W. H. Stockmayer, J. Chem. Phys. 36, 227 (1962).

[21] V. I. Abkevich, A. M. Gutin, and E. I. Shakhnovich, Foding \& Design, in press (1996).

[22] E. I. Shakhnovich and A. M. Gutin, Biophys. Chem. 34, 187 (1989).

[23] A. M. Gutin, V. I. Abkevich, and E. I. Shakhnovich, Proc.Natl.Acad.Sci 92, 1282 (1995).

[24] E.M.Lifshitz and L.P.Pitaevskii Physical Kinetics, Pergamon, London, 1981.

[25] N.Socci. J.Onuchic and P.Wolynes, J.Chem.Phys. 104, 5860 (1996).

[26] P.G.DeGennes Scaling Concepts in Polymer Physics. Cornell Univ. Press, Ithaca, NY, 1979.

[27] A. Sali, E. Shakhnovich, and M. Karplus, J. Mol. Biol. 235, 1614 (1994). 


\section{Figure Captions}

Fig.1 Lowest energy conformation for a random sequence of 40 amino acid residues.

Fig.2 Dependence of the folding time $t$ on the chain length $N$ for random sequences (black circles), for designed sequences (gray circles), and for the Go model (white circles). Folding time was estimated by averaging over 50 folding runs for each of the sequences.

Fig.3 Dependence of the folding time $t$ on the temperature $T$ for a random sequence of 40 residues with the ground state shown in Fig.1. Straight line approximates low-temperature part of the dependence. The slope of the line is the energy barrier $E_{b}$.

Fig.4 Dependence of the energy barrier $E_{b}$ on the energy of the native conformation $E_{n}$

for random sequences (black circles), for designed sequences (gray circles), and for the Go model (white circles). The line shows the best fit by $E_{b}=\phi\left|E_{n}\right|$ with $\phi=0.18$. 